\title{
Séroprévalence et facteurs de risque des hémoparasitoses (theilériose, babésiose et anaplasmose) chez les bovins dans quatre grandes régions d'élevage du Maroc
}

\author{
T. Rahali ${ }^{1 *}$ H. Sahibi ${ }^{1}$ A. Sadak ${ }^{2}$ S. Ait Hamou ${ }^{1}$ \\ B. Losson ${ }^{3}$ W.L. Goff ${ }^{4}$ A. Rhalem ${ }^{1}$
}

\begin{abstract}
Mots-clés
Bovin - Theilériose tropicale Babésiose - Anaplasmose - Facteur de risque - Maroc.
\end{abstract}

Résumé

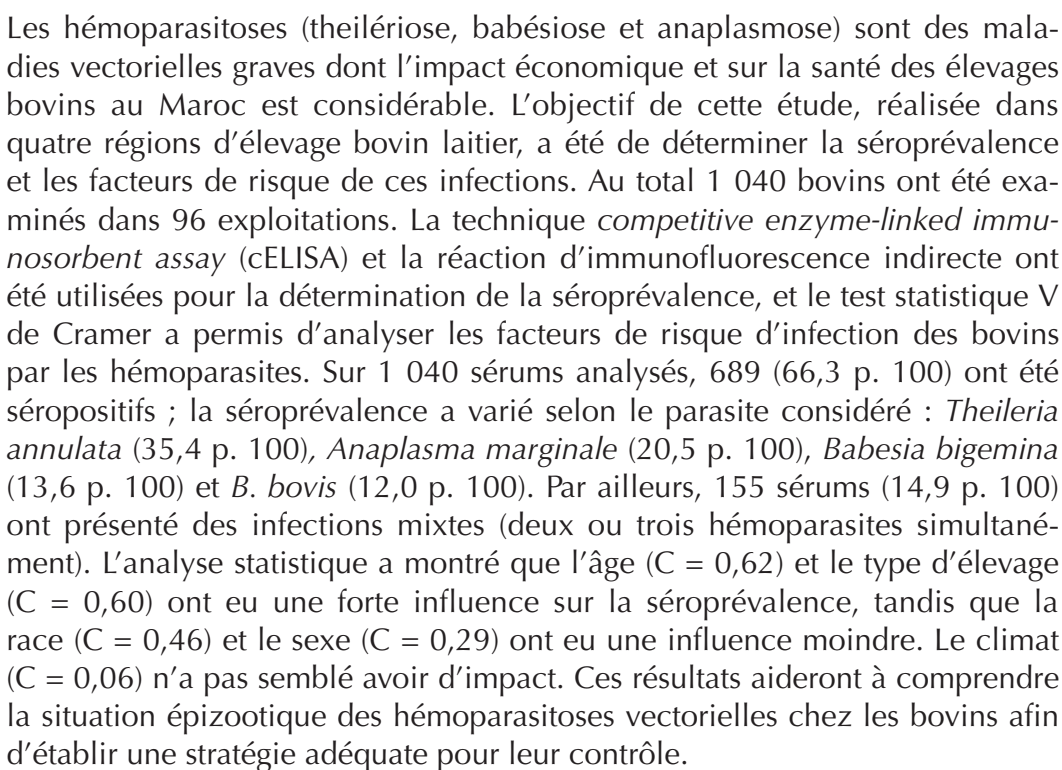

\section{INTRODUCTION}

Au Maroc, l'élevage des ruminants revêt une importance capitale dans l'activité économique rurale. Sa part dans le produit intérieur brut (PIB) agricole est estimée à 30 p. 100, soit 4,7 p. 100 du PIB global, et la taille du cheptel exploitée est estimée à 24 millions de têtes (2,7 millions de bovins, 16 millions d'ovins et 5 millions

\footnotetext{
1. Département de parasitologie et maladies parasitaires, Institut agronomique et vétérinaire Hassan II, BP 6202, Rabat-Instituts, Maroc.

2. Département de biologie générale, faculté des sciences, Université Mohammed V, Rabat, Maroc.

3. Laboratoire de parasitologie et maladies parasitaires, faculté de médecine vétérinaire, Université de Liège, 4000 Liège, Belgique.

4. Animal Disease Research Unit, Agricultural Research Service, US Department of Agriculture, Pullman, WA 99164-6630, USA

* Auteur pour la correspondance

Tél. : + 212618028213 ; e-mail : rahali.tarik@gmail.com
}

de caprins). C'est un secteur sur lequel la nouvelle stratégie agricole du Maroc, le « Plan Maroc Vert », mise pour la dynamisation de l'activité agricole. Ce plan préconise ainsi un développement important de la production laitière, avec une augmentation de la production de lait de 2,3 milliards de litres en 2011 à 5 milliards en $2020(3)$

Cependant, cet élevage est confronté à un sérieux problème qui limite son rendement et freine son développement. Il s'agit des maladies hémoparasitaires (theilériose, babésiose et anaplasmose) qui causent des dégâts considérables par la morbidité et la mortalité qu'elles occasionnent dans les cheptels. Ces maladies ont toujours existé au Maroc et ne représentaient pas de danger majeur pour les cheptels car ceux-ci bénéficiaient d'une situation épizootique stable, installée depuis plusieurs générations parmi les races animales locales. Mais leur gravité s'est considérablement accentuée suite à la politique d'importation de races étrangères, génétiquement plus performantes, entreprise dans les années 1970 dans 
le but d'améliorer le potentiel génétique du cheptel national. Ces animaux importés étaient dépourvus d'immunité contre les parasites locaux et succombaient rapidement aux infections. Ainsi, ces maladies sont passées, au cours de ces quatre dernières décennies, du statut de maladies quasiment inoffensives à celui de maladies qui représentent l'une des entraves majeures à l'élevage des ruminants.

Les pertes dues aux hémoparasitoses n'ont jamais été chiffrées de façon précise et à grande échelle, mais le nombre important de cas traités chaque année par les vétérinaires ainsi que la quantité impressionnante de médicaments hémoparasiticides utilisés (buparvaquone, imidocarb et tétracycline), selon les déclarations des vétérinaires et des éleveurs, témoignent de l'importance de ces maladies au Maroc (15). On a signalé ces dernières années des taux de mortalité dues à la theilériose allant jusqu'à 50 à 80 p. 100 chez les génisses pleines importées dans la région de Doukkala (14).

La présente étude fait partie d'un ambitieux programme de contrôle des tiques et des maladies transmises par les tiques au Maroc. Elle a eu pour objectif de connaître la situation épizootique actuelle de la theilériose bovine à Theileria annulata, des babésioses bovines à Babesia bovis et B. bigemina, et de l'anaplasmose bovine à Anaplasma marginale, en déterminant leurs séroprévalences et en analysant les facteurs de risque favorisant leur survenue dans les principales zones d'élevage au Maroc afin d'établir une stratégie de contrôle adéquate.

\section{MATERIEL ET METHODES}

\section{Régions d'étude}

La présente étude a été réalisée dans quatre régions d'élevage situées au centre nord-ouest du Maroc (figure 1). Ces quatre régions connaissent une grande concentration de bovins laitiers et $\underset{T}{ }$ fournissent à elles seules plus de 75 p. 100 de la production laiก่ tière du pays. Vingt-quatre sites ont été choisis dans chaque région

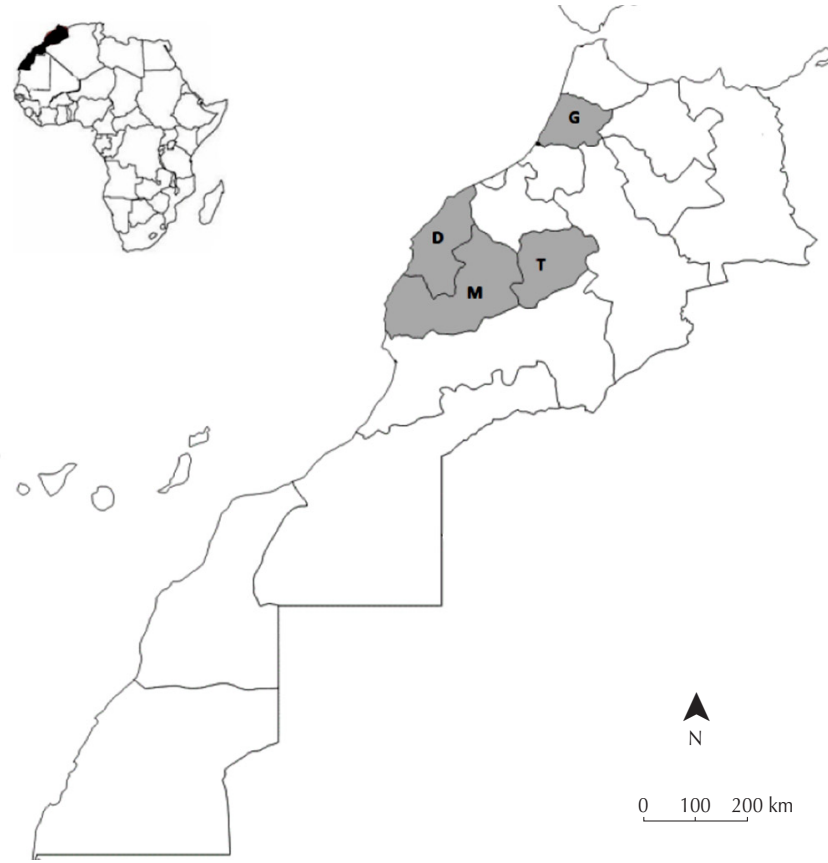

Figure 1 : situation géographique des régions concernées par l'étude. G : Gharb-Chrarda-Beni Hssen; D : Doukkala-Abda ; $T$ : Tadla-Azilal ; M : Marrakech-Tensift-Al Haouz. pour réaliser les prélèvements sur les bovins, et 96 exploitations ont ainsi été visitées.

\section{Région du Gharb-Chrarda-Beni Hssen}

Cette région de plaine s'étend sur $8805 \mathrm{~km}^{2}$ et sur deux étages bioclimatiques, l'un semi-aride, l'autre subhumide avec un hiver tempéré. Le climat y est de type méditerranéen, doux et humide en hiver, et chaud et sec en été. Les précipitations annuelles dépassent largement la moyenne nationale : elles sont partout supérieures à $600 \mathrm{~mm}$. La température moyenne varie entre 14 et $23{ }^{\circ} \mathrm{C}$. La moyenne des températures maximales du mois le plus chaud (août) est de $33{ }^{\circ} \mathrm{C}$ et la moyenne des températures minimales du mois le plus froid (janvier) est de $5{ }^{\circ} \mathrm{C}$. Le cheptel bovin est constitué de 433000 têtes dont 230000 sont destinées à la production laitière.

\section{Région de Doukkala-Abda}

Située au sud de la plaine centrale, sur la côte atlantique entre les deux grands fleuves Oum Rbia et Tensift, cette région s'étend sur $13285 \mathrm{~km}^{2}$. Elle s'inscrit dans le domaine du climat atlantique avec des températures qui restent clémentes en hiver et douces en été, aussi bien sur les côtes qu'à l'intérieur. Ces températures descendent rarement jusqu'à $0{ }^{\circ} \mathrm{C}$ en janvier et les maxima les plus fréquents de ce mois varient entre 14 et $18{ }^{\circ} \mathrm{C}$. En été, l'atmosphère se réchauffe sensiblement, les maxima les plus fréquents en juillet varient entre 16 et $26^{\circ} \mathrm{C}$. Le cheptel bovin est constitué de 410000 têtes dont 147000 sont destinées à la production laitière.

\section{Région de Tadla-Azilal}

Cette région s'étend sur $17125 \mathrm{~km}^{2}$, à une altitude moyenne de 400 à $700 \mathrm{~m}$ entre les plateaux de phosphate et le Moyen Atlas. Son climat est continental avec des précipitations variant entre 450 et $750 \mathrm{~mm}$ selon les années, qui la situent sur deux étages bioclimatiques méditerranéens, l'un semi-aride, l'autre subhumide. La moyenne des températures maximales du mois le plus chaud est de $39,8{ }^{\circ} \mathrm{C}$ et celle des températures minimales du mois le plus froid est de $3,9^{\circ} \mathrm{C}$. Le cheptel bovin de cette région est constitué de 182000 têtes dont 97000 sont destinées à la production laitière.

\section{Région de Marrakech-Tensift-Al Haouz}

Cette région s'étend sur $31160 \mathrm{~km}^{2}$. Le climat est en général aride ou semi-aride (altitude moyenne de $600 \mathrm{~m}$ ), mais humide dans l'Atlas (de 1500 m à $2000 \mathrm{~m}$ d'altitude) et sur le littoral. La pluviométrie annuelle est très variable : 50 p. 100 du territoire n'enregistrent que des niveaux inférieurs à $300 \mathrm{~mm}, 30$ p. 100 enregistrent une pluviométrie annuelle comprise entre 300 et $400 \mathrm{~mm}$ alors que 20 p. 100 du territoire, du côté de la chaine montagneuse du Haut-Atlas, enregistrent une pluviométrie supérieure à $400 \mathrm{~mm}$. La température moyenne de 80 p. 100 de la région est de $18{ }^{\circ} \mathrm{C}$ environ. La moyenne des températures maximales du mois le plus chaud est de $38,3{ }^{\circ} \mathrm{C}$ et la moyenne des températures minimales du mois le plus froid est de $4,5^{\circ} \mathrm{C}$. Le cheptel bovin est constitué de 400500 têtes dont 158000 sont destinées à la production laitière.

\section{Animaux et prélèvements sanguins}

Les prélèvements sanguins ont été effectués pendant le mois d'août 2008 sur 1040 bovins : 515 mâles et 525 femelles, 522 de races croisées et 518 de race locale, 508 âgés de moins d'un an et 532 de plus d'un an, 520 dans des élevages traditionnels et 520 dans des élevages modernes, et 780 dans un climat aride à semi-aride et 260 dans un climat semi-aride à subhumide. Les échantillons ont été prélevés au niveau de la veine jugulaire et mis dans des tubes secs sur lesquels ont été mentionnés l'âge, la race, le sexe, l'origine et le type d'élevage. Les sérums ont été ensuite récupérés et conservés à $-20^{\circ} \mathrm{C}$. 


\section{Analyses sérologiques}

Les analyses sérologiques ont été effectuées au laboratoire de parasitologie de l'Institut agronomique et vétérinaire Hassan II de Rabat, Maroc, au moyen de kits fournis par Animal Disease Research Unit, Agricultural Research Service, Department of Agriculture, Pullman, WA, USA.

Pour la recherche d'Anaplasma marginale, un test competitive enzyme-linked immunosorbent assay (cELISA) a été effectué selon les instructions du fabricant (13) qui a aussi fourni les contrôles positifs et négatifs.

Pour la recherche de Babesia bovis, un test cELISA, décrit par Goff et coll. (12) et permettant la recherche des anticorps spécifiques de B. bovis, ainsi que des plaques Mylar Pouch ont été utilisés. Les contrôles positif et négatif ont également été fournis.

Pour la recherche de Babesia bigemina, une technique basée sur l'immunofluorescence indirecte (IFI), décrite par Coons (8), a été utilisée pour révéler les anticorps anti-B. bigemina.

Pour la recherche de Theileria annulata, la révélation des anticorps anti-T. annulata a été effectuée à l'aide du test IFI décrit dans le manuel de diagnostic de l'OIE (18). Les étalements de schizontes de $T$. annulata ont été préparés selon la technique décrite par Pipano et Cahana (19), et Burridge (7).

\section{Analyse statistique}

Afin de déterminer la corrélation entre certains facteurs liés aux bovins (âge, sexe, race, type d'élevage et climat) et la séropositivité des échantillons, une analyse statistique des données a été réalisée en utilisant le test $\mathrm{V}$ de Cramer. Le $\mathrm{V}$ de Cramer $(\mathrm{C})$ est une forme centrée réduite du Chi deux. Il varie entre 0 et 1 . Plus la valeur de $\mathrm{C}$ est proche de 1 , plus il y a corrélation entre les deux variables.

\section{RESULTATS ET DISCUSSION}

Le taux d'infection des élevages par les hémoparasites a été très élevé dans toutes les régions de l'étude puisque 94 p. 100 des exploitations visitées étaient infectées par au moins un des quatre hémoparasites recherchés, et ainsi seulement 6 p. 100 étaient indemnes de ces parasites. En outre, la totalité des exploitations de type traditionnel était infectée, les séroprévalences ayant varié de 20 à 75 p. 100, ce qui témoignait de la présence de conditions favorables (climat, vecteurs, hôtes) à la prolifération et au maintien de ces infections.

Sur 1040 sérums de bovins analysés, 689 (66,3 p. 100) ont été positifs à l'un au moins des quatre hémoparasites étudiés (tableau I). L'infection à $T$. annulata a été la plus répandue avec une prévalence de 35,6 p. 100. Cette prédominance a déjà été rapportée au Maroc (1) et dans les pays voisins maghrebins, l'Algérie (30) et la Tunisie (6). Au Maroc, le vecteur naturel de T. annulata est la tique Hyalomma scupense (= Hyalomma detritum) qui est la tique dominante dans plusieurs régions d'élevage du pays $(21,22)$ ainsi que dans d'autres pays du Maghreb $(6,30)$.

L'infection à Babesia a été de 12 et 13,6 p. 100, respectivement pour $B$. bovis et $B$. bigemina. Les babésioses bovines et leurs vecteurs ont été rapportés dans plusieurs régions du Maroc $(1,4,10$, 24). Toutefois, les données épizootiques sur l'infection par Babesia varient d'une étude à l'autre. Sahibi et coll. (24) ont estimé la prévalence de $B$. bovis à 22 p. 100 au Gharb et 10 p. 100 à $\mathrm{Al} \mathrm{Haouz,}$ alors que nos résultats pour ces deux régions ont été respectivement de 6,9 et 8,8 p. 100. Pour B. bigemina, Sahibi et coll. (24) ont rapporté des prévalences de 10,8 p. 100 au Gharb et 0,0 p. 100 à $\mathrm{Al}$ Haouz alors que nos résultats ont révélé des prévalences respectives de 12,7 et 11,2 p. 100. La région d'Al Haouz était donc indemne, selon Sahibi et coll. (24), de B. bigemina en 1998. Ait Hammou et coll. (1) ont rapporté des séroprévalences dans la région du Gharb, respectivement pour $B$. bovis et $B$. bigemina de 23 et 25 p. 100, alors que nos résultats ont révélé des séroprévalences dans cette région de 6,9 p. 100 pour $B$. bovis et 12,7 p. 100 pour $B$. bigemina. Cette disparité dans les résultats pourrait s'expliquer en partie par la taille des échantillons, par les tests utilisés, mais probablement aussi par le fait que Rhipicephalus (Boophilus) annulatus, vecteur naturel de ce protozoaire, tique hygrophile, habituée au climat humide, modifie continuellement sa zone d'activité en suivant les réseaux d'irrigation, même dans les régions à climat aride, alors qu'une autre espèce, $R$. bursa, est suspectée de transmettre également cet hémoparasite aux bovins.

La séroprévalence globale de l'infection à $A$. marginale a été estimée à 20,5 p. 100 (tableau I). Les seules études sérologiques qui ont été réalisées pour estimer l'importance de cette infection au Maroc ont été conduites par Verhulst et coll. (26), Sahibi et coll. (25), et Ait Hammou et coll. (2), qui ont rapporté des

\section{Tableau I}

Séroprévalences des hémoparasitoses à Babesia bovis, B. bigemina, Theileria annulata et Anaplasma marginale selon la région de l'étude au Maroc

\begin{tabular}{|c|c|c|c|c|c|c|c|c|}
\hline \multirow[t]{2}{*}{ Région } & \multirow[t]{2}{*}{$\mathrm{Nb}$. fermes } & \multirow[t]{2}{*}{ Sérums analysés } & \multirow[t]{2}{*}{ Sérums positifs } & \multicolumn{5}{|c|}{ Séropositif (\%) } \\
\hline & & & & B. bovis & B. bigemina & T. annulata & A. marginale & $\begin{array}{l}\text { Infections } \\
\text { mixtes }\end{array}$ \\
\hline $\begin{array}{l}\text { Gharb-Chrarda- } \\
\text { Beni Hssen }\end{array}$ & 24 & 260 & $196(75,4)$ & $18(6,9)$ & $33(12,7)$ & $135(51,9)$ & $54(20,8)$ & $57(21,9)$ \\
\hline Doukkala-Abda & 24 & 260 & $178(68,5)$ & $57(21,9)$ & $39(15)$ & $74(28,5)$ & $70(26,9)$ & $44(16,9)$ \\
\hline Tadla-Azilal & 24 & 260 & $154(59,2)$ & $27(10,4)$ & $40(15,4)$ & $68(26,2)$ & $40(15,4)$ & $21(8,1)$ \\
\hline $\begin{array}{l}\text { Marrakech-Tensift- } \\
\text { Al Haouz }\end{array}$ & 24 & 260 & $161(61,9)$ & $23(8,8)$ & $29(11,2)$ & $93(35,8)$ & $49(18,8)$ & $33(12,7)$ \\
\hline Total & 96 & 1040 & $689(66,3)$ & $125(12,0)$ & $141(13,6)$ & $370(35,6)$ & $213(20,5)$ & $155(14,9)$ \\
\hline
\end{tabular}


séroprévalences respectivement de 9, 22,2 et 21,9 p. 100 dans différentes régions du Maroc. D'autres études dans des pays voisins rapportent des séroprévalences moins importantes, notamment 7,4 p. 100 en Algérie (30) ou et 3,5 p. 100 en Egypte (29).

Sur les 1040 sérums bovins analysés, 155 (14,9 p. 100) ont présenté des infections mixtes, soit deux ou trois hémoparasites à la fois $(B$. bovis $+B$. bigemina $; B$. bovis $+A$. marginale $; B$. bovis + T. annulata $;$ T. annulata + A. marginale $; B$. bovis $+B$. bigemina + A. marginale). De fait, nous avons constaté au niveau de la majorité des exploitations visitées un mélange et une proximité d'animaux domestiques divers (bovins, ovins, caprins, équins, volailles et lagomorphes), en plus des visiteurs sauvages (oiseaux et rongeurs), procurant une multitude d'hôtes potentiels pour différentes espèces de tiques vectrices de ces divers pathogènes. Ainsi, lors des collectes, plusieurs espèces de tiques ont été rencontrées au niveau d'une seule exploitation (quatre à cinq espèces par endroit) et sur un même animal (trois à quatre espèces). D'autres auteurs ont déjà rapporté ce phénomène au Maroc (23).

La séroprévalence des quatre hémoparasites étudiés a varié selon la région, pour $T$. annulata entre 26,2 et 51,9 p. 100 , pour $B$. bovis entre 6,9 et 21,9 p. 100, pour B. bigemina entre 11,2 et 15,4 p. 100, et pour $A$. marginale entre 15,4 et 26,9 p. 100 (tableau I). Ces taux indiquaient une instabilité enzootique au niveau des régions irriguées si on se réfère au modèle de Mahoney (16).

La région du Gharb a été la plus touchée par ces infections avec 75,4 p. 100 des bovins séropositifs (tableau I). Elle héberge un grand nombre de bovins et dispose d'un climat subhumide, d'un couvert végétal abondant, favorable à la survie et la prolifération des tiques, mais aussi des rongeurs et autres petits mammifères qui constituent les hôtes des stades immatures de certaines espèces, comme celles de Hyalomma.

Doukkala a été la deuxième région la plus touchée par ces infections avec 68,5 p. 100 de bovins séropositifs, suivie par $\mathrm{Al} \mathrm{Haouz}$ (61,9 p. 100), puis par Tadla (59,2 p. 100). Ces séroprévalences sont assez élevées malgré le fait que, contrairement à Gharb, ce sont des régions à climat aride ou semi-aride moins favorable à la survie et la prolifération des tiques. La seule explication plausible serait, à notre avis, la présence de microclimats humides favorisant la survie de ces ectoparasites. En effet, ces trois régions ont bénéficié de la mise en place d'un vaste réseau d'irrigation issu de grands barrages avec d'importantes retenues d'eau. Cela a adouci le climat de certaines zones arides et permis l'installation d'un couvert végétal abondant autour des retenues, assurant le gîte pour les tiques.

L'analyse statistique a montré que, parmi les facteurs influençant l'infection par les hémoparasites, l'âge de l'animal $(C=0,62)$ et le type d'élevage $(\mathrm{C}=0,60)$ ont eu une grande influence, tandis que la race $(C=0,46)$ et le sexe du bovin $(C=0,29)$ ont eu une influence moindre. Le climat $(C=0,06)$ n'a eu aucune influence significative (tableau II).

Concernant l'influence de l'âge sur l'infection des bovins par les hémoparasites, les bovins âgés de moins d'un an ont été moins sensibles à ces infections, comme déjà rapporté dans des études précédentes. Cela provient de la protection immunitaire innée qui s'estompe généralement après un an (28), et probablement aussi du fait que les animaux âgés peuvent davantage être infectés par les hémoparasites car ils sont exposés aux populations de tiques pendant plusieurs saisons. El Haj et coll. (10) ont observé que la proportion de bovins infectés augmentait avec l'âge.

Le type d'élevage a également eu une influence significative sur l'infection des bovins par les hémoparasites. Le nombre de bovins infectés a ainsi été plus important dans les élevages traditionnels

\section{Tableau II}

Influence du climat, du type d'élevage, de l'âge, du sexe et de l'origine des bovins sur l'infection des bovins par les hémoparasites dans le centre nord-ouest du Maroc

\begin{tabular}{lrrr} 
Facteurs de risque & Nb. animaux infectés (\%) & \multicolumn{2}{c}{ Tests } \\
\cline { 3 - 4 } & & \multicolumn{1}{c}{$\chi^{2}$} & C \\
\hline Age & & & \\
$<1$ an $(\mathrm{n}=508)$ & $48(9,4)$ & 665,70 & 0,62 \\
$>1$ an $(\mathrm{n}=532)$ & $476(89,5)$ & & \\
Sexe & & & \\
Mâle $(\mathrm{n}=515)$ & $268(52,0)$ & 92,15 & 0,29 \\
Femelle $(\mathrm{n}=525)$ & $421(80,2)$ & & \\
Race & & & \\
Locale $(\mathrm{n}=518)$ & $132(25,5)$ & 276,34 & 0,46 \\
Croisée $(\mathrm{n}=522)$ & $402(77,0)$ & & \\
Climat & & & \\
Subhumide/semi-aride $(\mathrm{n}=260)$ & $185(71,2)$ & 3,73 & 0,06 \\
Semi-aride/aride $(\mathrm{n}=780)$ & $504(64,6)$ & & \\
Type d'élevage & & & \\
Traditionnel $(\mathrm{n}=520)$ & $478(91,9)$ & 581,20 & 0,60 \\
Moderne $(\mathrm{n}=520)$ & $91(17,5)$ & &
\end{tabular}

que dans les élevages modernes. Ceci pourrait s'expliquer par la nature extensive du mode de pâturage, par le type de construction de ces exploitations qui représentent des gîtes favorables au développement des tiques endophiles vectrices de ces hémoparasites, et par le manque de traitements acaricides appropriés et réguliers.

La race a eu une influence moyennement significative sur l'infection des bovins par les hémoparasites, les bovins importés étant relativement plus sensibles que les bovins locaux. Enfin, le sexe a eu une influence peu significative sur l'infection des bovins par les hémoparasites. El Haj et coll. (10) ont rapporté que la proportion de séropositifs pour $B$. bigemina était plus importante chez les femelles, et Flach et coll. (11) ont rapporté que les bovins mâles étaient moins infectés par T. annulata que les femelles. Paradoxalement, le climat de la région n'a pas semblé avoir une influence significative sur l'infection par les hémoparasites $(C=0,06)$.

\section{- CONCLUSION}

Cette étude a montré que les hémoparasites bovins étudiés circulaient massivement dans les principales régions d'élevage bovin du Maroc. Les taux de contamination des élevages et des animaux étaient très élevés dans les quatre régions étudiées et un nombre important d'animaux étaient porteurs d'anticorps dirigés contre deux voire trois hémoparasites simultanément. La région du Gharb a été la plus infectée par ces pathogènes. Les anticorps dirigés contre la theilériose bovine à $T$. annulata ont été les plus fréquemment observés dans toutes les régions étudiées. Le type d'élevage, l'âge des animaux et la race ont été des facteurs favorisant la contamination par les hémoparasites. Cependant, d'après les séroprévalences relevées et malgré les taux d'infection élevés, la situation épizootique de ces maladies reste instable. 
Dans plusieurs pays du monde, la lutte contre les hémoparasitoses a été réalisée jusqu'à présent avec des moyens moins efficaces que ceux dont on dispose aujourd'hui et a surtout été basée sur la lutte contre les vecteurs au moyen d'acaricides. Des techniques sensibles et fiables d'immunologie moderne et de biologie moléculaire permettent désormais de lutter efficacement contre ces maladies. En raison des problèmes liés à l'application d'acaricides comme moyens de contrôle principaux, nous recommandons que les futures études soient centrées sur le développement de techniques basées sur la vaccination à l'aide de souches locales atténuées ou sur la méthode d'infection/traitement. La vaccination est utilisée chez les bovins contre la babésiose et la theilériose depuis plusieurs années, notamment en Israël (20), au Brésil (27), en Argentine (17) et en Australie (5). Les études réalisées dans les pays du Maghreb ont concerné la theilériose avec la mise au point d'un vaccin vivant, à base de lymphocytes infectés par des schizontes de T. annulata (Ouhelli et coll. [22] au Maroc, et Darghouth et coll. [9] en Tunisie).

\section{Remerciements}

Nous exprimons nos sincères remerciements aux vétérinaires et éleveurs des régions du Gharb, du Doukkala, du Tadla et d'Al Haouz pour leur aide précieuse sur le terrain. Nous tenons également à remercier les docteurs Abou $\mathrm{Al}$ Manadile, Ben Abdellah et Hachad pour leurs précieux conseils et leur assistance.

\section{BIBLIOGRAPHIE}

1. AIT HAMOU S., RAHALI T., SAHIBI H., BELGHYTI D., LOSSON B., RHALEM A., 2012. Séroprévalences des hémoparasitoses bovines dans deux régions irriguées du Maroc. Rev. Méd. Vét., $163:$ 480-485.

2. AIT HAMOU S., RAHALI T., SAHIBI H., BELGHYTI D., LOSSON B., GOFF W., RHALEM A., 2012. Molecular and serological prevalence of Anaplasma marginale in cattle of North Central Morocco. Res. Vet. Sci., 93: 1318-1323.

3. AUJOURD'HUI LE MAROC, 2013. Le Maroc récoltera les fruits du Plan Maroc Vert. www.aujourdhui.ma/une/carrefour-elevagebovin/\%C2\%AB-a-I-horizon-2020-le-maroc-recoltera-les-fruits-du-planmaroc-vert-\%C2\%BB-106217\#.VFi8FTTz2NY

4. BAILLY-CHOUMARA H., MOREL P.C., RAGEAU J., 1974. Première contribution au catalogue des tiques du Maroc (Acari, Ixodidae). Bull. Soc. Sci. Nat. Maroc, $\mathbf{5 4}:$ 71-80.

5. BOCK R.E., DE VOS A.J., 2001. Immunity following use of Australian tick fever vaccine: a review of the evidence. Aust. Vet. J., 79: 832-839.

6. BOUATTOUR A., 2001. Les tiques de Tunisie : rôle de Hyalomma detritum dans la transmission de Theileria annulata. Thèse Doct. Biologie, Faculté des sciences de Tunis, Tunisie, 247 p.

7. BURRIDGE M.J., 1971. Application of the indirect fluorescent antibody test in experimental East Coast fever (Theileria parva infection of cattle). Res. Vet. Sci., 12: 338-341.

8. COONS A.H., 1957. The application of fluorescent antibodies to the study of naturally occurring antibodies. Ann. N. Y. Acad. Sci., 69: 658662.

9. DARGHOUTH M.A., BOULTER N.R., GHARBI M., SASSI L., TAIT A., HALL R., 2006. Vaccination of calves with an attenuated cell line of Theileria annulata and the sporozoite antigen SPAG-1 produces a synergistic effect. Vet. Parasitol., 142: 54-62.

10. EL HAJ N., KACHANI M., OUHELLI H., BOUSLIKHANE M., AHAMI A.T., EL GUENNOUNI R., EL HASNAOUI M., KATENDE J.M., MORZARIA S.P., 2002. Etudes épidémiologiques sur Babesia bigemina au Maroc. Rev. Méd. Vét., 153: 809-814.

11. FLACH E.J., OUHELLI H., WADDINGTON D., OUDDICH M., SPOONER R.L., 1995. Factors influencing the transmission and incidence of tropical theileriosis (Theileria annulata infection of cattle) in Morocco. Vet. Parasitol., 59: 177-188.
12. GOFF W.L., MOLLOY J.B., JOHNSON W.C., SUAREZ C.E., PINO I., RHALEM A., SAHIBI H., CECI L., CARELLI G., ADAMS D.S., MCGUIRE T.C., KNOWLES D.P., MC ELWAIN T.F., 2006. Validation of a competitive enzyme-linked immunosorbent assay for detection of antibodies against Babesia bovis. Clin. Vaccine Immunol., 13: 1212-1216.

13. KNOWLES D.P., TORIONI DE ECHAIDE S., PALMER G.H., MCGUIRE T.C., STILLER D., MCELWAIN T.F., 1996. Antibody against an Anaplasma marginale MSP5 epitope common to tick and erythrocyte stages identifies persistently infected cattle. J. Clin. Microbiol., 34: 22252230.

14. MAHIN L., 2003. Etudes sur la maladie à Theileria annulata dans les Doukkala (Maroc). http://lucyin.walon.org/lv/theileria_papi.html

15. MAHIN L., 2006. Un forum sur la theilériose bovine dans les Doukkala (Maroc). http://lucyin.walon.org/lv/theileria_djauspinreye.html

16. MAHONEY D.F., 1977. Babesia in domestic animals. In: Kreier J.P., Ed., Parasitic protozoa, Vol. IV. New York, USA, Academic Press, p. 1-52.

17. MANGOLD A.J., VANZINI V.R., ECHAIDE I.E., DE ECHAIDE S.T., VOLPOGNI M.M., GUGLIELMONE A.A., 1996. Viability after thawing and dilution of simultaneously cryopreserved vaccinal Babesia bovis and Babesia bigemina strains cultured in vitro. Vet. Parasitol., 61: 345-348.

18. OIE, 2014. Manual of diagnostic tests and vaccines for terrestrial animals, chapter: 2.4.16. www.oie.int/en/international-standard-setting/ terrestrial-manual/access-online/

19. PIPANO E., CAHANA M., 1969. Fluorescent antibody test for the serodiagnosis of Theileria annulata. J. Parasitol., 55: 765.

20. PIPANO E., 1997. Vaccines against hemoparasitic diseases in Israel with special reference to quality assurance. Trop. Anim. Health Prod., 29 86S-90S.

21. OUHELLI H., FLACH E.J., 1992. Epidemiology and control of theileriosis in Morocco. In: Dolan T.T., Ed., Recent developments in the research and control of Theileria annulata. Proc. Workshop, ILRAD, Nairobi, Kenya, 17-19 Sept. 1990. Nairobi, Kenya, ILRAD, p. 19-20.

22. OUHELLI H., KACHANI M., FLACH E.J., WILLIAMSON S., EL HASNAOUI M., SPOONER R., 1997. Investigations on vaccination against theileriosis in Morocco. In: Hunter et al., Eds., Proc. Europ. Union Int. Symp. Ticks and Tick-Borne diseases, Xi'an, China, 3-6 Sept. 1996. Trop. Anim. Health Prod., 29: 103 S.

23. SAHIBI H., RHALEM A., 2007. Tiques et maladies transmises par les tiques chez les bovins au Maroc. Bulletin mensuel d'information et de liaison du PNTTA, 151 : 1-4.

24. SAHIBI H., RHALEM A., BERRAG B., GOFF W.L., 1998. Bovine babesiosis. Seroprevalence and ticks associated with cattle from two different regions of Morocco. Ann. N. Y. Acad. Sci., 849: 213-218.

25. SAHIBI H., RHALEM A., BERRAG B., GOFF W.L., 1998 Seroprevalence of bovine anaplasmosis in Morocco. Ann. N. Y. Acad. Sci., 849: 427-429.

26. VERHULST A., MAHIN L., THYS E., DE WITT K.J., 1983. Prevalence of antibodies to Anaplasma marginale in cattle from various African biotopes in central Morocco, north Cameroon and southeastern Zaïre. Zentralbl. Veterinarmed. B., 30: 537-540.

27. VIDOTTO O., BARBOSA C.S., ANDRADE G.M., MACHADO R.Z., DA ROCHA M.A., SILVA S.S., 1998. Evaluation of a frozen trivalent attenuated vaccine against babesiosis and anaplasmosis in Brazil. Ann. N. Y. Acad. Sci., 849: 420-423.

28. WOODFORD J.D., JONES T.W., RAE P.F., BOYD R., BELL-SAIKYI L., 1990. Sero-epidemiological studies of bovine babesiosis on Pemba Island, Tanzania. Vet. Parasitol., 37: 175-184.

29. YOUNIS E.E., HEGAZY N.A.M., EL-DEEB W., EL-KHATIB R.M., 2009. Epidemiological and biochemical studies on bovine anaplamosis in Dakahlia and Demiatta governorates in Egypt. Bull. Anim. Health Prod. Afr., 57: 297-309.

30. ZIAM H., BENAOUF H., 2004. Prevalence of blood parasites in cattle from wilayates of Annaba and El Tarf, East Algeria. Arch. Inst. Pasteur Tunis., 81: 27-30.

Accepted 23 January 2015; Online publication 30 September 2015 


\section{Summary}

Rahali T., Sahibi H., Sadak A., Ait Hamou S., Losson B., Goff W.L., Rhalem A. Seroprevalence and risk factors of bovine hemoparasitic diseases (theileriosis, babesiosis and anaplasmosis) in four major breeding areas of Morocco

Hemoparasitic diseases (theileriosis, babesiosis and anaplasmosis) are serious vector-borne diseases that have a considerable impact on cattle health and the farming economy of Morocco. The aim of this study, conducted in four dairy farming regions, was to determine the prevalence and risk factors for these infections. In total 1040 cattle were examined in 96 farms. The competitive enzyme-linked immunosorbent assay (cELISA) and fluorescent antibody techniques were used to determine the seroprevalence, and Cramer's $\vee$ statistical test was used to analyze risk factors for the infection of cattle by hemoparasites. Out of the 1040 analyzed sera, 689 (66.3\%) were positive. The seroprevalences varied depending on the parasite: Theileria annulata 35.4\%, Anaplasma marginale $20.5 \%$, Babesia bigemina 13.6\%, and B. bovis 12.0\%. Also 155 animals $(14.9 \%)$ had mixed infections with two or three hemoparasites simultaneously. The statistical analysis showed that the age $(C=0.62)$ and farming type $(C=0.60)$ had a high impact on animal infections, whereas the breed $(C=0.46)$ and gender $(C=0.29)$ had less impact. The climate $(C=0.06)$ did not seem to have any influence. Results of the present survey will help to understand the epizootical situation of vector-transmitted hemoparasitic diseases in cattle for the establishment of an adequate strategy for their control.

Keywords: Cattle - Tropical theileriosis - Babesiosis Anaplasmosis - Risk factor - Morocco.

\section{Resumen}

Rahali T., Sahibi H., Sadak A., Ait Hamou S., Losson B., Goff W.L., Rhalem A. Seroprevalencia y factores de riesgo de hemoparasitosis (theilerosis, babesiosis y anaplasmosis en los bovinos de cuatro regiones principales de cría de Marruecos

Las hemoparasitoses (theilerosis, babesiosis y anaplasmosis) son enfermedades vectoriales graves, cuyo impacto económico y sobre la salud de los criaderos bovinos en Marruecos es considerable. El objetivo del presente estudio, realizado en cuatro regiones de cría bovina lechera, fue de determinar la seroprevalencia y los factores de riesgo de estas infecciones. En total 1040 bovinos fueron examinados en 96 explotaciones. La técnica de inmunoensayo competitivo ligado a enzimas (cELISA) y la reacción de inmunofluorescencia se utilizaron épara la determinación de la seroprevalencia, y el test estadístico $V$ de Cramer permitió analizar los factores de riesgo de la infección en los bovinos por parte de las hemoparasitosis. De los 1040 sueros analizados, 689 (66,3\%) fueron positivos, la seroprevalencia varió según el parasito considerado: Theileria annulata (35,4\%), Anaplasma marginale $(20,5 \%)$, Babesia bigemina $(13,6 \%)$ y B. bovis $(12,0 \%)$. Por otro lado, 155 sueros (14,9\%) presentaron infecciones mixtas (dos o tres hemoparásitos simultáneamente). El análisis estadístico mostró que la edad $(C=0,62)$ y el tipo de cría $(C=0,60)$ ejercieron una gran influencia sobre la seroprevalencia, mientras que la raza $(C=0,46)$ y el sexo $(C=0,29)$ ejercieron una menor influencia. El clima $(C=0,06)$ no pareció tener fluencia sobre la infección de los bovinos por parte de los hemoparasitos. Estos resultados ayudaran a comprender la situación epizoótica de las hemoparasitosis vectoriales en los bovinos con la perspectiva del establecimiento de una estrategia adecuada para su control.

Palabras clave: Ganado bovino - Theileriosis tropical Babesiosis - Anaplasmosis - Factor de riesgo - Marruecos. 